DOI: $10.17516 / 1997-1397-2021-14-4-414-424$

УДК 517.95

\title{
The Regularity of the Solutions of Inverse Problems for the Pseudoparabolic Equation
}

\author{
Anna Sh. Lyubanova* \\ Siberian Federal University \\ Krasnoyarsk, Russian Federation
}

Received 10.02.2021, received in revised form 05.03.2021, accepted 20.04.2021

\begin{abstract}
The paper discusses the regularity of the solutions to the inverse problems on finding unknown coefficients dependent on $t$ in the pseudoparabolic equation of the third order with an additional information on the boundary. By the regularity is meant the continuous dependence of the solution on the input data of the inverse problem. The regularity of the solution is proved for two inverse problems of recovering the unknown coefficient in the second order term and the leader term of the linear pseudoparabolic equation.
\end{abstract}

Keywords: continuous dependence on the input data, a priori estimate, inverse problem, pseudoparabolic equation.

Citation: A.Sh. Lyubanova, The Regularity of the Solutions of Inverse Problems for the Pseudoparabolic Equation, J. Sib. Fed. Univ. Math. Phys., 2021, 14(4), 414-424.

DOI: 10.17516/1997-1397-2021-14-4-414-424.

\section{Introduction}

In this paper we discuss inverse problems for the pseudoparabolic diffusion equation

$$
(\nu u+\eta M u)_{t}+k M u+g u=f .
$$

Here $M$ is an elliptic linear differential operator of the second order in the space variables, $\nu \geqslant 0$ is a constant, the coefficients $\eta$ and $k$ depends on t, the functions $g$ and $f$ depends on $t, x$. We establish the regularity of the strong solution of two inverse problems for (0.1) with unknown coefficients $\eta$ and $k$ dependent on t under the Dirichlet boundary condition and additional integral boundary data akin to the conditions of overdetermination considered in $[5,6]$. An exact statement of the problems will be given below. In [6], the regularity of the strong solution was investigated for the inverse problem on finding an unknown coefficient $k(t)$ with given constant $\eta$ and function $g(t, x)$ in the sense that the smoothness of the solution increases with increasing the smoothness of the input data. In this paper by the regularity of the solution is meant its continuous dependence on the input data of the inverse problem. The regularity of solution, as used here, was established for the inverse problem of finding an unknown lower coefficient $g=g(t)$ in equation (0.1) [7].

In $[5,6]$, following the idea of $[9]$ the existence of the strong solutions of the inverse problems was proved by reducing the inverse problem to an operator equation of the second type for the

*lubanova@mail.ru

(C) Siberian Federal University. All rights reserved 
unknown coefficient. It was shown that the operator of this equation is a contraction on a set constructed with the use of the comparison theorems for pseudoparabolic equations.

Applications of such problems deal with the recovery of unknown parameters indicating physical properties of a medium (the heat conductivity, the permeability of a porous medium, the elasticity, the absorption (also known as potential) in the diffusion etc.). An exact statement of the problem will be given below. Since the natural stratum is involved, the parameters in (0.1) should be determined on the basis of the investigation of its behavior under the natural non-steady-state conditions (see $[1,12,13]$ for more details). This leads to the interest in studying the inverse problems for $(0.1)$ and its analogue.

The study of inverse problems for pseudoparabolic equations goes back to $1980 \mathrm{~s}$. The first result [11] refers to the inverse problems of determining a source function $f$ of equation

$$
\left(u+L_{1} u\right)_{t}+L_{2} u=f
$$

in case $L_{1}=L_{2}$ where $L_{1}$ and $L_{2}$ are the linear differential operators of the second order in spacial variables. We should mention also the results in $[2,8]$ concerning with coefficient inverse problems for the linear equation (0.1). In [8], the uniqueness theorem is obtained and an algorithm of determining the coefficients of $L_{2}$ is constructed. In [2], the solvability is established for two inverse problems of recovering the unknown coefficients in terms $u$ (the lowest term of $L_{2} u$ ) and $u_{t}$ of (0.2). In [10], an inverse problem of recovering time-depending right-hand side and coefficients of $(0.2)$ is considered. The values of the solution at separate points are employed as overdetermination conditions. The existence and uniqueness theorems are proven for this problem and the stability estimates of the solution are exposed.

The paper is organized as follows. Section 1 discusses the statement of the inverse problems. In Section 2 the regularity of the solution is investigated for the problem on recovering an unknown coefficient $k(t)$ in the second order term of the equation (0.1). Section 3 is devoted to the regularity of the solution to the problem on identification of the leader coefficient $\eta(t)$ in $(0.1)$.

\section{The statement of the problems}

Let $\Omega$ be a bounded domain in $\mathbf{R}^{n}$ with a boundary $\partial \Omega \in C^{2}, \bar{\Omega}$ be the closure of $\Omega$. T is an arbitrary real number, $Q_{T}=\Omega \times(0, T)$ with the lateral surface $S_{T}=(0, T) \times \partial \Omega, \bar{Q}_{T}$ is the closure of $Q_{T}$ and the pair $(t, x)$ is a point of $Q_{T}$.

From now on we keep the notations: $(\cdot, \cdot)_{R}$ is the inner product of $\mathbf{R}^{n} ;\|\cdot\|$ and $(\cdot, \cdot)$ are the norm and the inner product of $L^{2}(\Omega)$, respectively; $\|\cdot\|_{j}$ is the norm of $W_{2}^{j}(\Omega), j=1,2$; and $\langle\cdot, \cdot\rangle_{1}$ is the duality relation between $\stackrel{\circ}{W}_{2}^{j}(\Omega)$ and $W_{2}^{-j}(\Omega) ;\|\cdot\|_{p / 2}$ is the norm of $W_{2}^{p / 2}(\partial \Omega)$, $p=1,3$.

We introduce a linear differential operator $M=-\operatorname{div}(\mathcal{M}(x) \nabla)+m(x) I$ where $\mathcal{M}(x) \equiv$ $\left(m_{i j}(x)\right)$ is a matrix of functions $m_{i j}(x), i, j=1,2, \ldots, n ; I-$ the identity operator. We also keep the notation

$$
\left\langle M v_{1}, v_{2}\right\rangle_{M}=\int_{\Omega}\left(\left(\mathcal{M}(x) \nabla v_{1}, \nabla v_{2}\right)_{R}+m(x) v_{1} v_{2}\right) d x
$$

for $v_{1}, v_{2} \in W_{2}^{1}(\Omega)$ and assume that the following conditions are fulfilled. 
I. $m_{i j}(x), \partial m_{i j} / \partial x_{l}, i, j, l=1,2, \ldots, n$, and $m(x)$ are bounded in $\Omega$. Operator $M$ is elliptic, that is, there exist positive constants $m_{1}$ and $m_{2}$ such that for all $v \in W_{2}^{1}(\Omega)$

$$
m_{1}\|v\|_{1}^{2} \leqslant\langle M v, v\rangle_{M} \leqslant m_{2}\|v\|_{1}^{2} .
$$

II. $M$ is a selfadjoint operator, that is, $m_{i j}(x)=m_{j i}(x), i, j=1,2, \ldots, n$ for $x \in \Omega$.

In this paper we are studying the inverse problems of recovering unknown coefficients of the equation (0.1) with the initial data

$$
\left.(\nu u+\eta M u)\right|_{t=0}=U_{0}(x),
$$

and the boundary condition

$$
\left.u\right|_{\partial \Omega}=\beta(t, x) .
$$

We investigate the regularity of the solutions of two inverse problems.

Problem 1. For $\nu=1$, given functions $g(t, x), f(t, x), U_{0}(x), \beta(t, x), \omega(t, x), \varphi(t)$ and a constant $\eta$ find the pair of unknown functions $\{u, k\}, k=k(t)$, satisfying the equation (0.1), the initial data (1.2), the boundary condition (1.3) and the condition of overdetermination

$$
\int_{\partial \Omega}\left\{\eta \frac{\partial u_{t}}{\partial \bar{N}}+k \frac{\partial u}{\partial \bar{N}}\right\} \omega(t, x) d s+k \varphi_{1}(t)=\varphi_{2}(t) .
$$

Problem 2. For $\nu=0, k=1$, given functions $g(t, x), f(t, x), U_{0}(x), \beta(t, x), \omega(t, x), \varphi(t)$ and real constants $\mu_{1}, \mu_{2}$, find the pair of unknown functions $\{u, \eta\}, \eta=\eta(t)$, satisfying the equation (0.1), the initial data (1.2), the boundary condition (1.3) and the conditions of overdetermination

$$
\begin{gathered}
\int_{\partial \Omega}\left\{\left(\eta \frac{\partial u}{\partial \bar{N}}\right)_{t}+\frac{\partial u}{\partial \bar{N}}\right\} \omega(t, x) d s+\left(\eta \varphi_{1}(t)\right)_{t}=\varphi_{2}(t) \\
\eta(0) \int_{\partial \Omega} \frac{\partial u(0, x)}{\partial \bar{N}} \omega(0, x) d s+\mu_{1} \eta(0)=\mu_{2}
\end{gathered}
$$

Here $\frac{\partial}{\partial \bar{N}}=(\mathcal{M}(x) \nabla, \mathbf{n}), \mathbf{n}$ is the unit outward normal to the boundary $\partial \Omega$.

If $\omega \equiv 1$, then the integral conditions (1.5)-(1.6) means a given flux of a liquid through the surface $\partial \Omega$, for instance, the total discharge of a liquid through the surface of the ground. Similar nonlocal conditions were applied to control problems in [3].

We introduce functions $a(t, x), b(t, x)$ as the solutions of the Dirichlet problems

$$
\begin{gathered}
M a=0 \quad \text { in } \Omega,\left.\quad a\right|_{\partial \Omega}=\beta(t, x) ; \quad M b=0 \quad \text { in } \Omega,\left.\quad b\right|_{\partial \Omega}=\omega(t, x), \\
\Psi(t)=\langle M a, b\rangle_{1, M}, \quad F(t, x)=a_{t}-f(t, x)+g(t, x) a, \\
\bar{\Psi}=\max _{t \in[0, T]}\langle M a, b\rangle_{1, M}, \quad \bar{\varphi}_{1}=\max _{t \in[0, T]} \varphi_{1}(t),
\end{gathered}
$$

\section{The regularity of the solution to Problem 1}

By the strong solution of Problems 1 is meant the pair $\{u, k\} \in C^{1}\left([0, T] ; W_{2}^{2}(\Omega)\right) \times C([0, T])$ satisfying the equation (0.1) almost everywhere in $Q_{T}$ and the conditions (1.2)-(1.4) for almost all $(t, x) \in S_{T}$. 
In addition to the notations of Section 2 we introduce the function $h^{\eta}(t, x)$ as the solution of the Dirichlet problem

$$
h^{\eta}+\eta M h^{\eta}=0 \quad \text { in } \Omega,\left.\quad h^{\eta}\right|_{\partial \Omega}=\omega(t, x),
$$

and the notations

$$
\Phi^{\eta}(t)=\varphi_{2}(t)-\frac{\eta}{2}\left\langle M a_{t}, h^{\eta}\right\rangle_{1, M}+\left(f(t, x)-a_{t}, h^{\eta}\right), \quad \bar{\Phi}^{\eta}=\max _{t \in[0, T]} \Phi^{\eta}(t) .
$$

The existence and uniqueness of the strong solution to Problems 2 is established by the following theorem [6].

Theorem 2.1 Let the assumptions I-III be fulfilled and $\eta$ be a positive constant. Assume that (i) $f \in C\left([0, T] ; L^{2}(\Omega)\right), \beta \in C^{1}\left([0, T] ; W_{2}^{3 / 2}(\partial \Omega)\right), U_{0} \in L^{2}(\Omega), g \in C\left(\bar{Q}_{T}\right)$, $\omega \in C^{1}\left([0, T] ; W_{2}^{3 / 2}(\partial \Omega)\right), \varphi_{1} \in C^{1}([0, T]), \varphi_{2} \in C([0, T]) ;$

(ii) $f, U_{0}, \beta, \omega, \varphi_{1}$ are nonnegative and

$$
\int_{\Omega} h^{\eta} d x \geqslant h_{0}=\text { const }>0, \quad t \in[0, T]
$$

(iii) there exist positive constants $\alpha_{i}, i=0,1,2$, such that $\alpha_{0}, \alpha_{1} \leqslant 1, \alpha_{0}+\alpha_{1}<2$,

$$
\begin{gathered}
\left(1-\alpha_{0}\right) \varphi_{1}(t)+\left(1-\alpha_{1}\right) \Psi(t) \geqslant \alpha_{2}, \quad t \in[0, T], \\
\chi(0)+a(0, x)-U_{0}(x) \geqslant 0 \quad \text { for almost all } x \in \Omega, \\
g(t, x) \chi(t)+\chi^{\prime}(t)+F(t, x) \geqslant 0 \quad \text { for almost all }(t, x) \in Q_{T},
\end{gathered}
$$

where

$$
\chi(t)=\eta\left(\alpha_{0} \varphi_{1}(t)+\alpha_{1} \Psi(t)\right)\left[\int_{\Omega} h^{\eta} d x\right]^{-1}
$$

(iv) for any $t \in[0, T]$

$$
\Phi^{\eta}(t) \geqslant \Phi_{0}^{\eta}=\text { const }>0
$$

holds and $g(t, x)$ satisfies the inequality

$$
\max _{\bar{Q}_{T}} g(t, x) \leqslant \frac{\Phi_{0}^{\eta}}{\eta}\left[\bar{\varphi}_{1}+\bar{\Psi}+\eta^{-1} \max _{[0, T]}\left(a, h^{\eta}\right)\right]^{-1} \equiv \frac{k_{0}}{\eta} .
$$

Then Problem 1 has a unique solution $(u, k) \in C^{1}\left([0, T] ; W_{2}^{2}(\Omega)\right) \times C([0, T])$. Moreover, the estimates

$$
\begin{gathered}
0 \leqslant u(t, x) \leqslant \chi(t)+a(t, x) \quad \text { for almost all }(t, x) \in Q_{T}, \\
\|u\|_{2}^{2}+\left\|u_{t}\right\|_{2}^{2} \leqslant C_{1}, \quad t \in[0, T]
\end{gathered}
$$

are fulfilled and the coefficient $k(t)$ satisfies the inequalities

$$
K_{0} \leqslant k(t) \leqslant K_{1}
$$

with some positive constants $C_{1}, K_{0}$ and $K_{1}$.

In the hypotheses of Theorem 2.1 the strong solution of Problem 2 depends continuously on the input data of the problem. 
Theorem 2.2 Let the pair $\left\{u^{i}, k^{i}\right\}$ be the strong solution of Problem 1 with $\eta>0$ and the input data $\left\{f_{i}, g_{i}, \beta_{i}, U_{0}^{i}, \omega_{i}, \varphi_{1}^{i}, \varphi_{2}^{i}\right\}$ satisfying the hypotheses of Theorem 2.1, $i=1,2$. Then the estimates

$$
\begin{aligned}
& \|\tilde{k}\|_{C([0, T])} \leqslant C_{2}\left\{\frac{1}{\alpha_{2}}\left\|\tilde{\varphi}_{2}\right\|_{C([0, T])}+K_{1}\left\|\tilde{\varphi}_{1}\right\|_{C([0, T])}+\left\|\tilde{U}_{0}\right\|+\right. \\
& \left.+\max _{t \in[0, T]}\left[\|\tilde{f}\|+\|\tilde{\beta}\|_{W_{2}^{1 / 2}(\partial \Omega)}+\left\|\tilde{\beta}_{t}\right\|_{W_{2}^{1 / 2}(\partial \Omega)}+\|\tilde{g}\|_{C(\bar{\Omega})}+\|\tilde{\omega}\|_{W_{2}^{1 / 2}(\partial \Omega)}\right]\right\}, \\
& \|\tilde{u}\|_{C^{1}\left([0, T] ; W_{2}^{2}(\Omega)\right)} \leqslant C_{3}\left\{\frac{1}{\alpha_{2}}\left\|\tilde{\varphi}_{2}\right\|_{C([0, T])}+K_{1}\left\|\tilde{\varphi}_{1}\right\|_{C([0, T])}+\left\|\tilde{U}_{0}\right\|+\right. \\
& \left.\max _{t \in[0, T]}\left[\|\tilde{f}\|+\|\tilde{\beta}\|_{W_{2}^{3 / 2}(\partial \Omega)}+\left\|\tilde{\beta}_{t}\right\|_{W_{2}^{3 / 2}(\partial \Omega)}+\|\tilde{g}\|_{C(\bar{\Omega})}+\|\tilde{\omega}\|_{W_{2}^{1 / 2}(\partial \Omega)}\right]\right\},
\end{aligned}
$$

are valid for the difference $\{\tilde{u}, \tilde{k}\}=\left\{u^{1}-u^{2}, k^{1}-k^{2}\right\}$ with certain positive constants $C_{2}$ and $C_{3}$ where $\tilde{\varphi}_{j}=\varphi_{j}^{1}-\varphi_{j}^{2}, j=1,2, \tilde{U}_{0}=U_{0}^{1}-U_{0}^{2}, \tilde{\beta}=\beta_{1}-\beta_{2}, \tilde{f}=f_{1}-f_{2}, \tilde{g}=g_{1}-g_{2}, \tilde{\omega}=\omega_{1}-\omega_{2}$.

Proof. The difference $\{\tilde{u}, \tilde{k}\}$ obeys the relations

$$
\left\{\begin{array}{l}
\tilde{u}_{t}+\eta M \tilde{u}_{t}+k^{1}(t) M \tilde{u}+g_{1} \tilde{u}=\tilde{f}-\tilde{g} u^{2}-k M u^{2}, \\
\left.(\tilde{u}+\eta M \tilde{u})\right|_{t=0}=\tilde{U}_{0},\left.\quad \tilde{u}\right|_{S_{T}}=\tilde{\beta},
\end{array}\right.
$$

and the condition

$$
\int_{\partial \Omega}\left\{\frac{\partial}{\partial \bar{N}}\left[\eta \tilde{u}_{t}+k^{1} \tilde{u}+\tilde{k} u^{2}\right] \omega_{1}+\frac{\partial}{\partial \bar{N}}\left[\eta u_{t}^{2}+k^{2} u^{2}\right] \tilde{\omega}\right\} d S+\tilde{\varphi}_{1} k^{1}+\varphi_{1}^{2} \tilde{k}=\tilde{\varphi}_{2} .
$$

Multiplying the first equality of (2.7) by $\tilde{u}-\tilde{a}$ in terms of the scalar product of $L^{2}(\Omega)$, integration by parts in the second and third term of the left part and in the last term of the right side of the resulting relation gives

$$
\begin{aligned}
\frac{1}{2} \frac{\partial}{\partial t}\left(\|\tilde{u}-\tilde{a}\|^{2}+\right. & \left.\eta\langle M(\tilde{u}-\tilde{a}), \tilde{u}-\tilde{a}\rangle_{1}\right)+k^{1}(t)\|M(\tilde{u}-\tilde{a})\|^{2}+\left(g_{1}(\tilde{u}-\tilde{a}), \tilde{u}-\tilde{a}\right)= \\
= & (\tilde{f}, \tilde{u}-\tilde{a})-\left(\tilde{g} u^{2}, \tilde{u}-\tilde{a}\right)-\tilde{k}\left\langle M u^{2}, \tilde{u}-\tilde{a}\right\rangle_{1} .
\end{aligned}
$$

Integrating this equation with respect to $t$ on $(0, \tau), 0<\tau \leqslant T$, and estimating the right part with the help of (1.1), (2.2)-(2.4) and the Cauchy inequality one can obtain the estimate

$$
\begin{array}{r}
\|\tilde{u}-\tilde{a}\|^{2}+\eta\langle M(\tilde{u}-\tilde{a}), \tilde{u}-\tilde{a}\rangle_{1} \leqslant \int_{0}^{\tau}\left[\frac{2}{K_{0} m_{1}}\left(\|\tilde{f}\|^{2}+\left\|\tilde{a}_{t}\right\|^{2}+C_{1}\|\tilde{g}\|_{C(\bar{\Omega})}^{2}\right)+\frac{\bar{g}_{1}}{2}\|\tilde{a}\|^{2}\right] d t+ \\
+\left(\left\|\tilde{U}_{0}\right\|+\left\|\tilde{a}_{0}\right\|\right)^{2}+C_{1} m_{2} \int_{0}^{\tau}|\tilde{k}|^{2} d t
\end{array}
$$

where $\bar{g}_{1}=\left\|g_{1}\right\|_{c\left(\bar{Q}_{T}\right)}, \tilde{a}_{0}=\tilde{a}(0, x)$. Furthermore, multiplying the first equality of (2.7) by $M \tilde{u}$ in terms of the scalar product of $L^{2}(\Omega)$, integration by parts in the first term of the left part, integrating the result with respect to $t$ on $(0, \tau), 0<\tau \leqslant T$, and estimating the right part with the help of (1.1), (2.2)-(2.4), (2.9) and the Cauchy inequality we can get the estimate

$$
\begin{aligned}
\|\tilde{u}\|_{2} \leqslant C_{4}\left\{\int_{0}^{t}\left[\|\tilde{a}\|_{W_{2}^{2}(\Omega)}^{2}+\left\|\tilde{a}_{t}\right\|_{W_{2}^{2}(\Omega)}^{2}+\|\tilde{f}\|^{2}+\|\tilde{g}\|_{C(\bar{\Omega})}^{2}+|\tilde{k}|^{2}\right] d \tau\right\}^{1 / 2}+ \\
+\left\|\tilde{U}_{0}\right\|+c_{0}\|\tilde{a}\|_{W_{2}^{2}(\Omega)} .
\end{aligned}
$$


Here $c_{0}$ is the constant in the inequality

$$
\|\tilde{u}-\tilde{a}\|_{2} \leqslant c_{0}\|M \tilde{u}\|
$$

following from the second energy estimate for an elliptic operator [4, Ch. 2]; the constant $C_{4}>0$ depends on $\eta, m_{1}, m_{2}, K_{0}, K_{1}, T, C_{1},\left\|g_{i}\right\|_{C\left(\bar{Q}_{T}\right)}, i=1,2$. In a similar manner, multiplying the first equality of (2.7) by $M \tilde{u}_{t}$ in terms of the scalar product of $L^{2}(\Omega)$, integrating by parts in the first term of the left part, rearranging the third and fourth terms to the right side of the result we are led to the equation

$$
\left\langle\tilde{u}_{t}-\tilde{a}_{t}, M \tilde{u}_{t}\right\rangle_{1}+\eta\left\|M \tilde{u}_{t}\right\|^{2}=\left(-\tilde{a}_{t}-k^{1}(t) M \tilde{u}-g_{1} \tilde{u}+\tilde{f}-\tilde{g} u^{2}-k M u^{2}, M \tilde{u}_{t}\right)
$$

whence it follows by (1.1), (2.2)-(2.4), (2.9)-(2.11) and the Cauchy inequality that

$$
\begin{array}{r}
\left\|\tilde{u}_{t}\right\|_{2} \leqslant C_{5}\left\{\max _{t \in[0, T]}\left[\|\tilde{a}\|_{W_{2}^{2}(\Omega)}+\left\|\tilde{a}_{t}\right\|_{W_{2}^{2}(\Omega)}+\|\tilde{f}\|+\|\tilde{g}\|_{C(\bar{\Omega})}\right]+\left\|\tilde{U}_{0}\right\|+\right. \\
\left.+|\tilde{k}|+\left[\int_{0}^{t}|\tilde{k}|^{2} d \tau\right]^{1 / 2}\right\} .
\end{array}
$$

The positive constant $C_{5}$ depends on $\eta, m_{1}, m_{2}, K_{0}, K_{1}, T, C_{1}, C_{4}\left\|g_{i}\right\|_{C\left(\bar{Q}_{T}\right)}, i=1,2$.

On the other hand, as is shown in [6], following the idea of [9] we can reduce Problem 2 to an equivalent inverse problem with a nonlinear operator equation for $k^{i}(t)$. Really, let $h_{i}^{\eta}$ be the solution of the problem (2.1) with the boundary data $\omega_{i}$ instead of $\omega$. Multiplying (0.1) for $u^{i}, k^{i}$ by $h^{\eta}(t, x)$ in terms of the inner product in $L^{2}(\Omega)$, integrating by parts twice, substituting (1.6) into the resulting equation and taking into account (2.8) and the fact that

$$
\int_{\partial \Omega}\left(\eta \beta_{i t}+k^{i}(t) \beta_{i}\right) \frac{\partial h_{i}^{\eta}}{\partial \bar{N}} d s=-\eta\left\langle M a_{i t}, b^{i}\right\rangle_{M}-\left(a_{i t}, h_{i}^{\eta}\right)+k^{i}(t) \Psi^{i}(t)+\frac{k^{i}(t)}{\eta}\left(a_{i}, h_{i}^{\eta}\right),
$$

we obtain

$$
k^{i}(t)\left(\varphi_{1}^{i}(t)+\Psi^{i}(t)+\frac{1}{\eta}\left(a_{i}-u^{i}, h_{i}^{\eta}\right)\right)=\Phi_{i}^{\eta}(t)-\left(g_{i} u^{i}, h_{i}^{\eta}\right), \quad i=1,2,
$$

where $\Psi^{i}(t)=\left\langle M a_{i}, b_{i}\right\rangle_{1, M}, \Phi_{i}^{\eta}(t)=\varphi_{2}^{i}(t)-\frac{\eta}{2}\left\langle M a_{i t}, b_{i}\right\rangle_{1, M}+\left(f_{i}-a_{i t}, h_{i}^{\eta}\right)$, the functions $a_{i}$ and $b_{i}$ are the solutions of the problems (1.7) with the boundary data $\beta_{i}$ and $\omega_{i}$ instead of $\beta$ and $\omega$, respectively.

Setting up the difference of the operator equations (2.13) for $i=1$ and $i=2$ we are led to the equation

$$
\begin{gathered}
\tilde{k}(t)\left(\varphi_{1}^{1}+\Psi_{1}+\frac{1}{\eta}\left(a_{1}-u^{1}, h_{1}^{\eta}\right)\right)=\tilde{\Phi}^{\eta}-\left(g_{1} \tilde{u}, h_{1}^{\eta}\right)-\left(\tilde{g} u^{2}, h_{1}^{\eta}\right)- \\
-\left(g_{2} u^{2}, \tilde{h}^{\eta}\right)-k^{2}\left(\tilde{\varphi}_{1}+\tilde{\Psi}+\frac{1}{\eta}\left(\tilde{a}-\tilde{u}, h_{1}^{\eta}\right)+\frac{1}{\eta}\left(a_{2}-u^{2}, \tilde{h}^{\eta}\right)\right),
\end{gathered}
$$

where $\tilde{a}=a_{1}-a_{2}, \tilde{\Phi}^{\eta}=\Phi_{1}^{\eta}-\Phi_{2}^{\eta}, \tilde{h}^{\eta}=h_{1}^{\eta}-h_{2}^{\eta}, \tilde{\Psi}=\Psi_{1}-\Psi_{2}$. Estimating the right side of this equation with the use of $(2.2)-(2.4),(2.9)$ one can obtain the inequality

$$
\begin{aligned}
|\tilde{k}| \leqslant C_{6}\left[\left\|\tilde{a}_{t}\right\|_{1}+\|\tilde{a}\|+\|\tilde{b}\|_{1}+\left\|\tilde{h}^{\eta}\right\|+\|\tilde{f}\|+\|\tilde{g}\|_{C(\bar{\Omega})}+\left\|\tilde{U}_{0}\right\|+\left\|\tilde{a}_{0}\right\|\right]+ & \\
& +\frac{1}{\alpha_{2}}\left(\left|\tilde{\varphi}_{2}\right|+K_{1}\left|\tilde{\varphi}_{1}\right|\right)+C_{7} \int_{0}^{\tau}|\tilde{k}|^{2} d t
\end{aligned}
$$


where positive constants $C_{6}, C_{7}$ depends on $K_{0}, K_{1}, \eta, T, m_{1}, m_{2}, C_{1},\left\|g_{i}\right\|_{C(\bar{\Omega})}, \bar{\varphi}_{1}$, $\max _{t \in[0, T]}\left\{\left\|a_{i}\right\|,\left\|a_{i t}\right\|,\left\|b_{i}\right\|,\left\|f_{i}\right\|\right\}, i=1,2$. By Gronwall's lemma and the inequality

$$
\|v\|_{j} \leqslant c_{j}\|v\|_{W_{2}^{j-1 / 2}(\partial \Omega)}
$$

valid for all $v \in W_{2}^{j}(\Omega)$ and an integer $j \geqslant 1$ (see [4, Ch. 2]), (2.14) implies (2.5). Now the estimate (2.6) follows from (2.5), (2.10), (2.12) and (2.15). Theorem is proved.

\section{The regularity of the solution to Problem 2}

By the strong solution of Problem 2 is meant the pair $\{u, \eta\} \in C^{1}\left([0, T] ; W_{2}^{2}(\Omega)\right) \times C^{1}([0, T])$ satisfying the equation (0.1) almost everywhere in $Q_{T}$ and the conditions (1.2), (1.3), (1.5), (1.6) for almost all $(t, x) \in S_{T}$.

The existence and uniqueness of the strong solution to Problem 2 is established by the following theorem [5].

Theorem 3.1 Let the assumptions I-II be fulfilled and $\partial \Omega \in C^{2}$. Assume that

i) $f \in C\left([0, T] ; L^{2}(\Omega)\right), \beta \in C^{1}\left([0, T] ; W_{2}^{3 / 2}(\partial \Omega)\right), U_{0} \in L^{2}(\Omega), g \in C\left(\bar{Q}_{T}\right)$, $\omega \in C^{1}\left([0, T] ; W_{2}^{3 / 2}(\partial \Omega)\right), \varphi_{1} \in C^{1}([0, T]), \varphi_{2} \in C([0, T]) ;$

ii) $f, U_{0}, \beta, \omega$ and $\varphi_{1}$ are nonnegative, $g \leqslant 0, \mu_{2}>0$ and $\varphi_{1}(0)=\mu_{1}$;

iii) $\Psi(t) \geqslant 0$ and there exist a positive constant $\alpha$ such that

$$
\begin{gathered}
\varphi_{1}(t)+\Psi(t) \geqslant \alpha, \quad t \in[0, T], \\
\Phi(t) \equiv \varphi_{2}(t)-\Psi(t)+(f, b) \geqslant 0 .
\end{gathered}
$$

Then Problem 2 has a unique solution $\{u, \eta\}$ in the class

$$
V=\left\{\{u, \eta\} \mid u \in C^{1}\left([0, T] ; W_{2}^{2}(\Omega)\right), \eta \in C^{1}([0, T])\right\} .
$$

Moreover, there are positive constants $\eta_{0}$ and $\eta_{1}$ such that for all $t \in[0, T]$

$$
\eta_{0} \leqslant \eta(t) \leqslant \eta_{1}
$$

and the estimates

$$
\begin{gathered}
\left|\eta^{\prime}\right| \leqslant C_{8}, \\
\|u\|_{2}+\left\|u_{t}\right\|_{2} \leqslant C_{9}
\end{gathered}
$$

holds with certain constants $C_{8}$ and $C_{9}$.

In the hypotheses of Theorem 3.1 the strong solution of Problem 2 depends continuously on the input data of the problem.

Theorem 3.2 Let the pair $\left\{u^{i}, \eta^{i}\right\}$ be the strong solution of Problem 2 with the input data $\left\{f_{i}, g_{i}, \beta_{i}, U_{0}^{i}, \omega_{i}, \varphi_{1}^{i}, \varphi_{2}^{i}, \mu_{1}^{i}, \mu_{2}^{i}\right\}$ satisfying the hypotheses of Theorem 3.1, $i=1,2$. Then the estimates

$$
\begin{aligned}
& \|\tilde{\eta}\|_{C^{1}([0, T])} \leqslant C_{10}\left\{\left\|\tilde{\varphi}_{1}\right\|_{C^{1}([0, T])}+\left\|\tilde{\varphi}_{2}\right\|_{C([0, T])}+\left\|\tilde{U}_{0}\right\|+\|\tilde{g}\|_{C\left(\bar{Q}_{T}\right)}+\left|\tilde{\mu}_{2}\right|+\right. \\
& \left.\quad+\max _{t \in[0, T]}\left[\|\tilde{f}\|+\|\tilde{\beta}\|_{W_{2}^{1 / 2}(\partial \Omega)}+\left\|\tilde{\beta}_{t}\right\|_{W_{2}^{1 / 2}(\partial \Omega)}+\|\tilde{\omega}\|_{W_{2}^{1 / 2}(\partial \Omega)}+\left\|\tilde{\omega}_{t}\right\|_{W_{2}^{1 / 2}(\partial \Omega)}\right]\right\},
\end{aligned}
$$




$$
\begin{aligned}
& \|\tilde{u}\|_{C^{1}\left([0, T] ; W_{2}^{2}(\Omega)\right)} \leqslant C_{11}\left\{\left\|\tilde{\varphi}_{1}\right\|_{C^{1}([0, T])}+\left\|\tilde{\varphi}_{2}\right\|_{C([0, T])}+\left\|\tilde{U}_{0}\right\|+\|\tilde{g}\|_{C(\bar{\Omega})}+\left|\tilde{\mu}_{2}\right|+\right. \\
& \left.\quad+\max _{t \in[0, T]}\left[\|\tilde{f}\|+\|\tilde{\beta}\|_{W_{2}^{3 / 2}(\partial \Omega)}+\left\|\tilde{\beta}_{t}\right\|_{W_{2}^{3 / 2}(\partial \Omega)}+\|\tilde{\omega}\|_{W_{2}^{1 / 2}(\partial \Omega)}+\left\|\tilde{\omega}_{t}\right\|_{W_{2}^{1 / 2}(\partial \Omega)}\right]\right\},
\end{aligned}
$$

are valid for the difference $\{\tilde{u}, \tilde{\eta}\}=\left\{u^{1}-u^{2}, \eta^{1}-\eta^{2}\right\}$ with certain positive constants $C_{10}$ and $C_{11}$ where again $\tilde{\varphi}_{j}=\varphi_{j}^{1}-\varphi_{j}^{2}, j=1,2, \tilde{U}_{0}=U_{0}^{1}-U_{0}^{2}, \tilde{\beta}=\beta_{1}-\beta_{2}, \tilde{f}=f_{1}-f_{2}, \tilde{g}=g_{1}-g_{2}$, $\tilde{\omega}=\omega_{1}-\omega_{2}$.

Proof. The difference $\{\tilde{u}, \tilde{\eta}\}$ obeys the relations

$$
\left\{\begin{array}{l}
\left(\eta_{1} M \tilde{u}\right)_{t}+M \tilde{u}+g_{1} \tilde{u}=\tilde{f}-\tilde{g} u^{2}-\left(\tilde{\eta} M u^{2}\right)_{t}, \\
\left.\left(\eta_{1} M \tilde{u}\right)\right|_{t=0}=\tilde{U}_{0}-\left.\left(\tilde{\eta} M u^{2}\right)\right|_{t=0},\left.\quad \tilde{u}\right|_{S_{T}}=\tilde{\beta},
\end{array}\right.
$$

and the conditions

$$
\begin{gathered}
\int_{\partial \Omega}\left\{\frac{\partial}{\partial \bar{N}}\left[\left(\eta^{1} \tilde{u}\right)_{t}+\left(\tilde{\eta} u^{2}\right)_{t}+\tilde{u}\right] \omega_{1}+\frac{\partial}{\partial \bar{N}}\left[\left(\eta^{2} u^{2}\right)_{t}+u^{2}\right] \tilde{\omega}\right\} d s+\left(\tilde{\eta} \varphi_{1}^{1}\right)_{t}+\left(\eta^{2} \tilde{\varphi}_{1}\right)_{t}=\tilde{\varphi}_{2}, \\
\left.\int_{\partial \Omega}\left\{\frac{\partial}{\partial \bar{N}}\left[\eta^{1} \tilde{u}+\tilde{\eta} u^{2}\right] \omega_{1}+\eta^{2} \frac{\partial u^{2}}{\partial \bar{N}} \tilde{\omega}\right\} d s\right|_{t=0}+\tilde{\mu}_{1} \eta^{1}(0)+\mu_{1}^{2} \tilde{\eta}(0)=\tilde{\mu}_{2} .
\end{gathered}
$$

Multiplying the first equality of (3.4) by $\exp \left(\int_{0}^{t}\left(\eta^{1}(\tau)\right)^{-1} d \tau\right)$, integration with respect to $t$ from 0 to $\theta, 0<\theta \leqslant T$, and solving the resulting equation for $M \tilde{u}$ gives

$$
\begin{array}{r}
M \tilde{u}=\frac{1}{\eta^{1}(\theta)}\left\{\tilde{U}_{0} \exp \left(-\int_{0}^{\theta} \frac{d \tau}{\eta^{1}(\tau)}\right)+\int_{0}^{\theta}\left(\tilde{f}-g_{1} \tilde{u}-\tilde{g} u_{2}\right) \exp \left(-\int_{t}^{\theta} \frac{d \tau}{\eta^{1}(\tau)}\right) d t-\right. \\
\left.-\tilde{\eta} M u^{2}+\int_{0}^{\theta} \frac{\tilde{\eta}}{\eta^{1}(t)} M u^{2} \exp \left(-\int_{t}^{\theta} \frac{d \tau}{\eta^{1}(\tau)}\right) d t\right\} .
\end{array}
$$

Furthermore, multiplying this equation by $\tilde{u}-\tilde{a}$ in terms of the inner product of $L^{2}(\Omega)$, integrating by parts in the left side of the resulting equation and estimating the right one with (3.2), (3.4) one can obtain the inequality

$$
\|\tilde{u}\|_{1} \leqslant C_{12}\left\{\|\tilde{a}\|_{C\left([0, T] ; W_{2}^{1}(\Omega)\right)}+\left\|\tilde{U}_{0}\right\|+\int_{0}^{T}\left(\|\tilde{f}\|+\|\tilde{g}\|_{C(\bar{\Omega})}\right) d t+\left(\int_{0}^{t}|\tilde{\eta}|^{2} d \theta\right)\right\}
$$

where the constant $C_{12}>0$ depends on $m_{1}, T, c_{0},\left\|g_{1}\right\|_{C\left(\bar{Q}_{T}\right)}$ and the constant $m_{3}>0$ from the inequality

$$
\|M v\|_{2} \leqslant m_{3}\|v\|_{2}
$$

valid for all $v \in W_{2}^{2}(\Omega)$. Now we estimate the left and right sides of (3.8) in the norm of $L^{2}(\Omega)$ with the use of (2.11), (3.2), (3.4). Taking into account (3.10) we get

$$
\begin{aligned}
\|\tilde{u}\|_{2} \leqslant\|\tilde{a}\|_{2}+c_{0}\|M \tilde{u}\| \leqslant\|\tilde{a}\|_{2}+C_{13}\left\{\left\|\tilde{U}_{0}\right\|+\int_{0}^{\theta}\left(\|\tilde{f}\|+\|\tilde{g}\|_{C(\bar{\Omega})}\right) d t+|\tilde{\eta}|+\int_{0}^{\theta}|\tilde{\eta}| d t\right\}+ \\
+\bar{g}_{1} \int_{0}^{\theta}\|\tilde{u}\|_{2} d t
\end{aligned}
$$

whence in accordance with Gronwall's lemma

$$
\|\tilde{u}\|_{2} \leqslant C_{14}\left\{\|\tilde{a}\|_{C\left([0, T] ; W_{2}^{2}(\Omega)\right)}+\left\|\tilde{U}_{0}\right\|+\int_{0}^{T}\left(\|\tilde{f}\|+\|\tilde{g}\|_{C(\bar{\Omega})}\right) d t+\int_{0}^{t}|\tilde{\eta}| d \theta\right\} .
$$


Here the positive constants $C_{13}$ and $C_{14}$ depends on $\eta_{0}, T, m_{3}, c_{0},\left\|g_{1}\right\|_{C\left(\bar{Q}_{T}\right)}$.

On the other hand, it is shown in [6] that following the idea of [9] one can reduce Problem 2 to an equivalent inverse problem with a nonlinear operator equation for $\eta^{i}(t)$. Really, let us multiply (2.4) by $b$ in terms of the inner product of $L^{2}(\Omega)$ and integrate by parts twice in the second and third terms. In view of (1.5)-(1.7) we have

$$
\frac{d}{d t}\left(\eta^{i}\left(\varphi_{1}^{i}+\Psi^{i}\right)\right)-\eta^{i}\left\langle M a_{i}, b_{i t}\right\rangle_{1}=\Phi_{i}-\left(g_{i} u^{i}, b_{i}\right)
$$

where $\Phi_{i}=\Phi_{i}(t) \equiv \varphi_{2}^{i}(t)-\Psi_{i}(t)+\left(f_{i}, b_{i}\right)$, the functions $a_{i}$ and $b_{i}$ are again the solutions of the problems (1.7) with the boundary data $\beta_{i}$ and $\omega_{i}$ instead of $\beta$ and $\omega$, respectively. By (1.5), multiplying this equation by $\zeta_{i}(t) \equiv \exp \left(-\int_{0}^{t}\left\langle M a_{i}, b_{i \tau}\right\rangle_{1}\left(\varphi_{1}^{i}+\Psi_{i}\right)^{-1} d \tau\right)$ and integration with respect to $t$ from 0 to $\theta, 0<\theta \leqslant T$ gives

$$
\eta^{i}(\theta)\left(\varphi_{1}^{i}(\theta)+\Psi_{i}(\theta)\right) \zeta_{i}(\theta)=\eta^{i}(0)\left(\varphi_{1}^{i}(0)+\Psi_{i}(0)\right)+\int_{0}^{\theta}\left[\Phi_{i}-\left(g_{i} u^{i}, b_{i}\right)\right] \zeta_{i} d t, \quad i=1,2 .
$$

Furthermore, we multiply the second relation in (3.7) by $b_{i}(0, x)=b_{i}^{0}(x)$ in terms of the inner product of $L^{2}(\Omega)$ and integrate by parts twice in the resulting equation. Taking into account (1.3) for $t=0$ and (1.6), we obtain

$$
\eta^{i}(0)\left(\mu_{1}^{i}+\Psi_{i}(0)\right)=\mu_{2}^{i}+\left(U_{0}^{i}, b_{i}^{0}\right), \quad i=1,2 .
$$

Substituting (3.13) into the operator equation (3.12) and setting up the difference of the resulting equations for $i=1$ and $i=2$ we are led to the equality

$$
\begin{array}{r}
\tilde{\eta}(\theta)\left(\varphi_{1}^{1}(\theta)+\Psi_{1}(\theta)\right) \zeta_{1}(\theta)=-\eta^{2}(\theta)\left[\left(\tilde{\varphi}_{1}(\theta)+\tilde{\Psi}(\theta)\right) \zeta_{1}(\theta)+\left(\varphi_{1}^{2}(\theta)+\Psi_{2}(\theta)\right) \tilde{\zeta}(\theta)\right]+ \\
+\tilde{\mu}_{2}+\left(\tilde{U}_{0}, b_{1}^{0}\right)+\left(U_{0}^{2}, \tilde{b}^{0}\right)+\int_{0}^{\theta}\left[\tilde{\Phi}-\left(\tilde{g} u^{1}, b_{1}\right)-\left(g_{2} \tilde{u}, b_{1}\right)-\left(g_{2} u^{2}, \tilde{b}\right)\right] \zeta_{1} d t+ \\
+\int_{0}^{\theta}\left[\Phi_{2}-\left(g_{2} u^{2}, b_{2}\right)\right] \tilde{\zeta} d t
\end{array}
$$

where $\tilde{\zeta}=\zeta_{1}-\zeta_{2}, \tilde{b^{0}}=b_{1}^{0}-b_{2}^{0}, \tilde{\Phi}=\Phi_{1}-\Phi_{2}$. Let $y_{i}(t)=\int_{0}^{t}\left\langle M a_{i}, b_{i \tau}\right\rangle_{1}\left(\varphi_{1}^{i}+\Psi_{i}\right)^{-1} d \tau, i=1,2$. By (3.1) and the definition of functions $\zeta, a$ and $b$,

$$
|\tilde{\zeta}|=\left|\int_{y_{1}(t)}^{y_{2}(t)} e^{-y} d y\right| \leqslant \exp \left(\max _{i=1,2}\left|y_{i}(t)\right|\right)\left|y_{1}-y_{2}\right| \leqslant C_{15} \int_{0}^{t}\left[\|\tilde{a}\|_{1}+\left\|\tilde{b}_{\tau}\right\|_{1}+\left|\tilde{\varphi}_{1}\right|+|\tilde{\Psi}|\right] d \tau .
$$

The constant $C_{15}$ depends on $m_{2},\left\|\varphi_{1}^{i}\right\|_{C([0, T])}, \max _{t \in[0, T]}\left\{\left\|a_{i}\right\|,\left\|b_{i}\right\|,\left\|b_{i t}\right\|\right\}, i=1,2$. Estimating the right side of the equation (3.14) with regard to (3.2)-(3.4), (3.9) and (3.15) one can obtain the inequality

$$
\begin{gathered}
|\tilde{\eta}| \leqslant C_{16}\left\{\left\|\tilde{\varphi}_{1}\right\|_{C([0, T])}+\|\tilde{a}\|_{C\left([0, T] ; W_{2}^{1}(\Omega)\right)}+\|\tilde{b}\|_{C^{1}\left([0, T] ; W_{2}^{1}(\Omega)\right)}+\left|\tilde{\mu}_{2}\right|+\left\|\tilde{U}_{0}\right\|+\right. \\
\left.+\left\|\tilde{\varphi}_{2}\right\|_{C([0, T])}+\|\tilde{f}\|_{L^{2}\left(Q_{T}\right)}+\|\tilde{g}\|_{C\left(\bar{Q}_{T}\right)}\right\}+C_{17} \int_{0}^{t}|\tilde{\eta}| d \tau
\end{gathered}
$$


which implies by Gronwall's lemma that

$$
\begin{array}{r}
|\tilde{\eta}| \leqslant C_{16} e^{C_{17} T}\left\{\left\|\tilde{\varphi}_{1}\right\|_{C([0, T])}+\|\tilde{a}\|_{C\left([0, T] ; W_{2}^{1}(\Omega)\right)}+\|\tilde{b}\|_{C^{1}\left([0, T] ; W_{2}^{1}(\Omega)\right)}+\left|\tilde{\mu}_{2}\right|+\left\|\tilde{U}_{0}\right\|+\right. \\
\left.+\left\|\tilde{\varphi}_{2}\right\|_{C([0, T])}+\|\tilde{f}\|_{L^{2}\left(Q_{T}\right)}+\|\tilde{g}\|_{C\left(\bar{Q}_{T}\right)}\right\} .
\end{array}
$$

Here the positive constants $C_{16}$ and $C_{17}$ depend on $\eta_{0}, \eta_{1}, T, m_{2}, C_{9} C_{14}, C_{15},\left\|g_{i}\right\|_{C\left(\bar{Q}_{T}\right)}$, $\left\|\varphi_{1}^{i}\right\|_{C([0, T])}, \max _{t \in[0, T]}\left\{\left\|a_{i}\right\|,\left\|a_{i t}\right\|,\left\|b_{i}\right\|,\left\|b_{i t}\right\|,\left\|f_{i}\right\|\right\}, i=1,2$.

We are now in a position to obtain the estimates for $\tilde{\eta}^{\prime}$ and $\tilde{u}_{t}$. Solving the first equation of (3.7) for $M \tilde{u}_{t}$ and estimating the right side of the resulting equation with the use of (2.11), (3.2)-(3.4), (3.10), (3.11) yields

$$
\left\|\tilde{u}_{t}\right\|_{2} \leqslant\left\|\tilde{a}_{t}\right\|_{2}+C_{18}\left\{\left\|\tilde{U}_{0}\right\|+\max _{t \in[0, T]}\left\{\|\tilde{a}\|_{2}+\|\tilde{f}\|+|\tilde{\eta}|\right\}+\|\tilde{g}\|_{C\left(\bar{Q}_{T}\right)}+\left|\tilde{\eta}^{\prime}\right|\right\}
$$

where the constant $C_{18}>0$ depends on $m_{3}, \eta_{0}, T, c_{0}, C_{8}, C_{9}, C_{14},\left\|g_{1}\right\|_{C\left(\bar{Q}_{T}\right)}$. Furthermore, differentiating (3.14) and estimating the right part of the resulting equation with (3.2)-(3.4), (3.9) and (3.15) we are led to the relation

$$
\begin{aligned}
\left|\tilde{\eta}^{\prime}\right| & =\mid-\tilde{\eta}\left[\left(\left(\varphi_{1}^{1}\right)^{\prime}+\left(\Psi_{1}\right)^{\prime}\right) \zeta_{1}+\left(\varphi_{1}^{1}+\Psi_{1}\right) \zeta_{1}^{\prime}\right]-\left(\eta^{2}\right)^{\prime}\left[\left(\tilde{\varphi}_{1}+\tilde{\Psi}\right) \zeta_{1}+\left(\varphi_{1}^{2}+\Psi_{2}\right) \tilde{\zeta}\right]- \\
& -\eta^{2}\left[\left(\tilde{\varphi}_{1}^{\prime}+\tilde{\Psi}^{\prime}\right) \zeta_{1}+\left(\left(\varphi_{1}^{2}\right)^{\prime}+\Psi_{2}^{\prime}\right) \tilde{\zeta}+\left(\tilde{\varphi}_{1}+\tilde{\Psi}\right) \zeta_{1}^{\prime}+\left(\varphi_{1}^{2}+\Psi_{2}\right) \tilde{\zeta}^{\prime}\right]+\left(\Phi_{2}-\left(g_{2} u^{2}, b_{2}\right)\right) \tilde{\zeta}+ \\
+ & {\left[\tilde{\Phi}-\left(\tilde{g} u^{1}, b_{1}\right)-\left(g_{2} \tilde{u}, b_{1}\right)-\left(g_{2} u^{2}, \tilde{b}\right)\right] \zeta_{1} \mid\left(\left(\varphi_{1}^{1}+\Psi_{1}\right) \zeta_{1}\right)^{-1} \leqslant C_{19}\left[\left\|\tilde{\varphi}_{1}\right\|_{C^{1}([0, T])}+\left\|\tilde{U}_{0}\right\|+\right.} \\
& \left.+\left\|\tilde{\varphi}_{2}\right\|_{C([0, T])}+\|\tilde{g}\|_{C\left(\bar{Q}_{T}\right)}+\max _{t \in[0, T]}\left\{\|\tilde{f}\|+\|\tilde{a}\|_{1}+\left\|\tilde{a}_{t}\right\|_{1}+\|\tilde{b}\|_{1}+\left\|\tilde{b}_{t}\right\|_{1}\right\}+\left|\tilde{\mu}_{2}\right|\right]
\end{aligned}
$$

Here the positive constants $C_{19}$ depends on $\eta_{0}, \eta_{1}, T, m_{2}, C_{9} C_{14}, C_{15}, C_{16}, C_{17},\left\|g_{i}\right\|_{C\left(\bar{Q}_{T}\right)}$, $\left\|\varphi_{1}^{i}\right\|_{C^{1}([0, T])}, \max _{t \in[0, T]}\left\{\left\|a_{i}\right\|,\left\|a_{i t}\right\|,\left\|b_{i}\right\|,\left\|b_{i t}\right\|,\left\|f_{i}\right\|\right\}, i=1,2$. By (2.15), the inequalities (3.16) and (3.18) imply (3.5). Now the estimate (3.6) follows from (2.15), (3.11), (3.16)-(3.18).

\section{References}

[1] G.I.Barenblatt, Iu.P.Zheltov, I.N.Kochina, Basic concepts in the theory of seepage of homogeneous liquids in fissured blocks [strata], J. Appl. Math. Mech., 24(1960), 1286-1303 (in Russian).

[2] A.I.Kozhanov, O razreshimosti koeffitsientnikh obratnikh zadach dlya nekotorikh uravnenii sobolevskogo tipa (On the solvability of the coefficient inverse problems for equations of Sobolev type), Nauchniye vedomosti Belgorodskogo gosudarstvennogo universiteta. Seriya "Matematika. Phizika", 2010, no. 5, 88-98 (in Russian).

[3] T.-T.Li, L.W.White, Total Flux (Nonlocal) Boundary Value Problems for Pseudoparabolic Equation, Appl. Anal., 16(1983), 17-31.

[4] O.A.Ladyzhenskaya, V.A.Solonnikov, N.N.Ural'ceva, Linear and Quasilinear Equations of Parabolic Type. Transl. Math. Mono., vol. 23, AMC, Providence, 1968; English transl. Nauka, Moskva, 1967. 
[5] A.Sh.Lyubanova, Inverse Problem for a Pseudoparabolic Equation with Integral Overdetermination Conditions, Differential Equations, 50(2014), 502-512.

DOI: $10.1134 /$ S0012266114040089

[6] A.Sh.Lyubanova, A.Tani, An inverse problem for pseudoparabolic equation of filtration. The existence, uniqueness and regularity, Appl. Anal., 90(2011), 1557-1571.

DOI: $10.1080 / 00036811.2010 .530258$

[7] A.Sh.Lyubanova, A.V.Velisevich, Inverse problems for the stationary and pseudoparabolic equations of diffusion, Appl. Anal., 98(2019), 1997-2010.

DOI: $10.1080 / 00036811.2018 .1442001$

[8] M.Sh.Mamayusupov, O zadache opredeleniya koeffitsiyentov psevdoparabolicheskogo uravneniya (The problem of determining coefficients of a pseudoparabolic equation), Studies in integro-differential equations, Ilim, Frunze, 1983, no. 16, 290-297 (in Russian).

[9] A.I.Prilepko, D.G.Orlovsky, I.A.Vasin, Methods for solving inverse problems in mathematical physics, New York, Marcel Dekker, 2000.

[10] S.G.Pyatkov, S.N.Shergin, On some mathematical models of filtration type. Bulletin of the South Ural State University. Ser. Mathematical Modelling, Programming and Computer Software (Bulletin SUSU MMCS), 2015, no. 8, 105-116.

[11] W.Rundell, Determination of an unknown nonhomogeneous term in a linear partial differential equation from overspecified boundary data, Appl. Anal., 10(1980), 231-242.

[12] R.E.Showalter, T.W.Ting, Pseudoparabolic partial differential equations, SIAM J. Math. Anal., 1(1970), 1-26.

[13] A.G Sveshnikov, A.B.Alshin, M.O.Korpusov, Yu.D.Pletner, Lineyniye i nelineyniye uravneniya sobolevskogo tipa (Linear and nonlinear equations of the Sobolev type), Physmatlit, Moskow, 2007 (in Russian).

\title{
Регулярность решений обратных задач для псевдопарабо- лических уравнений
}

Анна Ш. Любанова

Сибирский федеральный университет Красноярск, Российская Федерация

\begin{abstract}
Аннотация. В работе обсуждается регулярность решений обратных задач отыскания неизвестного коэффициента, зависящего от времени, в псевдопараболическом уравнении третьего порядка по дополнительной информации о решении на границе. Доказана регулярность решения двух обратных задач восстановления неизвестного коэффициента в члене второго порядка и старшем члене линейного псевдопараболического уравнения.
\end{abstract}

Ключевые слова: непрерывная зависимость от исходных данных, априорная оценка, обратная задача, псевдопараболическое уравнение. 